\title{
Changing the Paradigm of Cancer Control in Cuba
}

\section{By Teresa Romero, MD, MS}

Cuba's National Cancer Registry records 29,000 new cases of cancer annually, some $50 \%$ of which are among persons living in the western provinces, and $55 \%$ among persons aged $\geq 65$ years. [1] Cancer in Cuba is associated with a rapidly aging population and risk factors linked to certain social determinants of health-both social conditions and lifestyles. At current rates of growth, incidence is projected to reach 30,000 cases by 2010 .

Despite significant development of Cuba's universal public health system, national cancer programs, biomedical research, and oncology and related medical fields, cancer incidence and mortality continue to climb in the Cuban population of 11.2 million. This contrasts with generally decreasing adjusted mortality from heart and cerebrovascular disease-the other killers in the top-mortality triad. If these trends continue, cancer will soon become the number one cause of death in Cuba, a status it already claims in 8 of the country's 14 provinces.[1]

Over 20,000 persons died from cancer in Cuba in 2008.[2] Some $60 \%$ of cancer mortality is concentrated in 7 sites: lung, prostate, female breast, colon-rectum, hematopoietic system, stomach and pancreas. Cancer is the first cause of years of potential life lost (YPLL), 6 years more than for heart disease.[3] The mortality/incidence ratio for some cancer sites is as high as 0.60 ; cervical cancer mortality is increasing; adjusted prostate cancer mortality stands at 24.6;[2] and smoking prevalence remains over $30 \%$.[4]

Such unacceptable indicators prompted a re-evaluation of the health system's cancer control efforts in 2004, pinpointing several problems contributing to the continued rise of cancer incidence and mortality:

- Equipment and institutional infrastructure were depleted by the economic crisis of the 1990s.

- While data were available on cancer incidence and mortality, they had not been used to generate a sufficiently profound evaluation of the complexities of cancer in Cuba as an evidence base for policy-making.

- Cancer initiatives and services did not adequately respond to real needs at different levels and in specific locales; flexibility and agility were lacking in response to need.

- Resources were not being used most efficiently, requiring better identification, prioritization and coordination.

- Programs and initiatives were fragmented, lacking clear and coordinated leadership. There was no single entity responsible for national cancer control in all its facets.

- Cancer control did not effectively reach beyond the public health sector to critical participants in other sectors and in the general public.

This assessment led to the creation of the National Cancer Control Unit (UNCC, its Spanish acronym) in 2006,[5] responsible for all cancer control efforts. Its first job was to initiate an overhaul of public health strategies to reach the goal of reducing cancer mortality $15 \%$ by 2015 and thus contribute to increasing life expec-

\author{
Cuban Cancer Care, Research and Control (1961-2008) \\ 1961 - Oncology Hospital of Havana established \\ - Oncology Section of the Ministry of Public Health \\ established \\ - First national cancer control plan devised \\ 1964 - National Cancer Registry established \\ 1966 - Oncology Hospital becomes the National Oncology \\ and Radiobiology Institute (INOR) for oncology \\ development, cancer care, policy and research \\ - Therapeutic Norms for Cancer Care published \\ - Technical training programs begun in radiotherapy \\ 1968 - Oncology established as an official medical specialty \\ - National Oncology Group created \\ 1969 - Experimental Chemotherapy Service set up at INOR \\ 1986 - National Cancer Registry's Information System \\ improved, including mandatory nationwide reporting of \\ all new cases \\ 1987 - First National Cancer Control Program implemented \\ 1992 - Program for Reducing Cancer Mortality reorganized \\ 1994 - Molecular Immunology Center (CIM) established \\ 1999 - Anti-Smoking Prevention and Control Program initiated \\ - Public Education Program in Cancer implemented \\ 2004 - National Cancer Research Programs developed (by \\ cancer type) \\ 2006 - National Cancer Control Unit established \\ 2006-2008 - Comprehensive Cancer Control Program reorganized
}

tancy and quality of life for the Cuban population. The result was the Comprehensive Cancer Control Program (PICC, its Spanish acronym), now in effect throughout Cuba.

The Program's purpose is to "develop and pursue a strategic action plan that propitiates broad alliances to control cancer; evaluates the cancer burden by main sites, gender, and age groups in order to determine priorities; and designs and implements action plans that effectively respond to the complexities of the problem and its specific geographic, social and economic characteristics. The aim is to reduce incidence and mortality, as well as improve survival rates and quality of life for cancer patients, using national and international experiences as standards for reference."[2]

Ministerial Resolution 49 establishing the UNCC charged it with the following additional responsibilities:[5]

- Establish new organizational forms for cancer control.

- Control efficient use of human and material resources.

- Formulate guidelines for development of human resources.

- Achieve multidisciplinary and intersectoral capabilities, nationally and locally, with flexible needs-based action plans.

- Analyze current information and develop new data sources to contribute to evidence-based decision-making.

- Systematically control screening, diagnosis, treatment, rehabilitation and palliative care, based on best practices standards to improve patient-centered care.

- Propose national information, education and social communication strategies.

- Establish research priorities and participate in research planning by cancer site and type, facilitating clinical trials and generalized introduction of results. 
- Coordinate with the Scientific Pole (biotechnology) and other institutions.

- Evaluate cancer control program initiatives and results at all levels, through inspections and other means.

- Generate evidence-based proposals for investments and new projects, monitor their implementation, and ensure rational use of resources.

The UNCC functions as a coordinating body for all cancer control policies and actions throughout the country, both in and outside the health sector. Its organizational model relies on knowledge management and innovation, focusing much of its energies on improving scientific analysis for decision-making (data gathering, processing and evaluation), facilitating innovative solutions to specific problems identified through action research, and reducing the time needed to translate results into action and policy.

Through the upgraded PICC_-bringing all initiatives under a single national umbrella-the UNCC prioritizes work in four areas nationwide:

1. Seven types of cancer: lung, female breast, prostate, colorectal, uterine cervix, oral cavity and childhood cancers. Although the latter accounts for only $2 \%$ of total cancer deaths, it is included as a priority due to its social importance and sensitivity.

2. Development and control of cancer therapies and resources, including radiotherapy, clinical and surgical oncology, and nuclear medicine, as well as application of natural and traditional medicine and biotechnology products.

\section{Social determinants associated with cancer}

4. Broad participation in cancer control (other governmental and social sectors, mass media and the public).

The PICC extends to provinces, municipalities and communities through the UNCC's national networks and designated personnel at all levels. At the national level, the UNCC itself is organized into working groups on: PICC coordination and operations; the National Cancer Registry; Science and Technology (innovation and evaluation); and the Cancer Observatory (broad-based data gathering and relevant knowledge sharing). Key to the UNCC's coordinating function is its relationship with biotechnology research institutions, the National Oncology and Radiobiology Institute (INOR), the National Oncology Group and the newly-created Social Sciences and Humanities Council. Some 23 expert groups involving nearly 400 professionals also collaborate with the UNCC.

The National Cancer Control Network and its branches-Observatory, Cancer Registry, and Best Practices-link all the groups and institutions involved in cancer control throughout the island through INFOMED, the health system's electronic communication platform.

At the provincial, municipal and community levels, there are, for the first time, professionals designated to lead and coordinate cancer control efforts, as well as organize cancer teams at all three levels, which also interface with institutions outside the health system. As of May 2009, all these positions have been filled and personnel given special training.
Cancer Mortality Reduction Goals for 2015

\begin{tabular}{|c|c|c|}
\hline Objective: Reduce cancer mortality by $\mathbf{1 5 \%}$ \\
\hline & \multicolumn{3}{|c|}{ Goals } \\
\hline Baseline & For $\mathbf{2 0 1 0}$ & For $\mathbf{2 0 1 5}$ \\
\hline 115.3 & $7 \%$ & $15 \%$ \\
\hline 115.3 & 107.2 & 98 \\
\hline
\end{tabular}

Indicator: Adjusted mortality per 100,000 population

Source: Ministry of Public Health, National Statistics Division, Havana.

\begin{tabular}{|c|c|c|c|}
\hline \multicolumn{4}{|c|}{$\begin{array}{l}\text { Objective: Reduce lung cancer mortality by } 8 \% \\
\text { Goals }\end{array}$} \\
\hline & Baseline & For 2010 & For 2015 \\
\hline & & $4 \%$ & $8 \%$ \\
\hline General & 27.3 & 26.2 & 25.1 \\
\hline Men & 38.6 & 37.0 & 35.3 \\
\hline Women & 17.0 & 16.3 & 15.6 \\
\hline
\end{tabular}

Indicator: Adjusted mortality by sex per 100,000 population

Source: Ministry of Public Health,National Statistics Division, Havana, 2004.

\begin{tabular}{|c|c|c|}
\hline \multicolumn{3}{|c|}{ Objective: Reduce cervical cancer mortality by $20 \%$} \\
\hline Baseline & For 2010 & Goals \\
\hline 5.3 & $10 \%$ & $20 \%$ \\
\hline 5.3 & 4.7 & 4.2 \\
\hline
\end{tabular}

Indicator: Adjusted mortality per 100,000 population

Source: Ministry of Public Health, National Statistics Division, Havana, 2004

\begin{tabular}{|l|c|c|c|}
\multicolumn{4}{|c|}{ Objective: Reduce colon cancer mortality by $\mathbf{8 \%}$} \\
\hline & Baseline & For $\mathbf{2 0 1 0}$ & Foals $\mathbf{2 0 1 5}$ \\
\hline General & & $4 \%$ & $8 \%$ \\
\hline Men & 9.4 & 9.0 & 8.6 \\
\hline Women & 9.5 & 9.1 & 8.7 \\
\hline
\end{tabular}

Indicator: Adjusted mortality by sex per 100,000 population

Source: Ministry of Public Health, National Statistics Division, Havana, 2004.

\begin{tabular}{|c|c|c|}
\hline Objective: Reduce breast cancer mortality in women by $\mathbf{1 5 \%}$ \\
\hline & \multicolumn{3}{|c|}{ Goals } \\
\hline Baseline & For $\mathbf{2 0 1 0}$ & For $\mathbf{2 0 1 5}$ \\
\hline 14.1 & $7 \%$ & $15 \%$ \\
\hline 14.1 & 13.1 & 11.9 \\
\hline
\end{tabular}

Indicator: Adjusted mortality per 100,000 population

Source: Ministry of Public Health, National Statistics Division, Havana, 2004.

\begin{tabular}{|c|c|c|}
\hline Objective: Reduce prostate cancer mortality by $\mathbf{8} \%$ \\
\hline Baseline & For 2010 & Foals 2015 \\
26.0 & $4 \%$ & $8 \%$ \\
\hline 26.0 & 25.0 & 23.9 \\
\hline
\end{tabular}

Indicator: Adjusted mortality per 100,000 population

Source: Ministry of Public Health, National Statistics Division, Havana, 2004.

Translated from the report Proyecciones de la salud pública en Cuba para el 2015 (Public Health Projections in Cuba for Year 2015)

Emphasis is currently placed on organizing cancer control at the local level, involving the PICC chiefs in each of the country's nearly 500 community-based polyclinics-covering virtually the entire population. These physicians are responsible for ensuring quality care for cancer patients, including diagnosis and patient followup, accurate reporting of cancer cases to the National Registry, assuring $100 \%$ screening of risk groups, coordination of local training, monitoring of worker and patient satisfaction, and promotion of intersectoral initiatives, among other aspects. Their functions may also be extended, as determined by analysis of local social determinants, epidemiological stratification, and mapping of needs and resources. 
Two of their most challenging tasks are, 1) seeing to it that all health institutions are smoke-free and 2) setting up local cancer control teams, each of which involves a psychologist, rehabilitation specialist, social worker, internist, oncologist, pediatrician, dentist, and other health professionals as needed.

Each year, the National Cancer Assembly brings together representatives of each province to evaluate the previous year's results and propose the plan of action for the following year, including changes in national strategies. While it is too soon to assess the impact of these paradigm changes on cancer trends in Cuba, the 2008 Assembly noted several encouraging developments:

- Budgets for the Program's logistical support have increased during the last three years, facilitating national coverage of essential cancer medications.

- The Program's leadership is functioning with coordinators on the job at all levels, including all provinces and municipalities.

- All polyclinics now have chiefs of comprehensive cancer care who direct the Program's actions at the community level, having received post-graduate training for their new role.

- $\quad$ Specialized care for children with cancer has been concentrated in 9 hospitals with the necessary resources to provide national coverage and meet ISO-9000 norms. (INOR is already certified and the other 8 hospitals are in process of certification.)

- Technological development in radiotherapy and nuclear medicine has proceeded with the acquisition of new equipment.

- Plans for research on the most common cancer sites, begun in 2004 , have been completed.

- The national mass media strategy has been redesigned.

Several important conceptual changes have been introduced in the practice of cancer control in Cuba, coinciding with World Health Organization recommendations-the implications of which should be documented and evaluated over time:

1. Implement a single national cancer control program, as opposed to a variety of fragmented sub-programs.

2. Start cancer control not with screening for disease but with health-promotion of healthy living, focusing on "protective factors" rather than just "risk factors".
3. Re-orient services to be more patient-centered, taking into account each patient's particular circumstances and environment.

4. Promote an intersectoral approach; cancer is too complex, with many determinants, to be the responsibility of the health sector alone.

5. Broaden data collection to generate a more detailed picture of cancer trends and epidemiology, and to better measure results of national and local actions.

6. Make full use of the virtual network connectivity provided by INFOMED.

7. Achieve more rational development and distribution of resources based on scientific evidence of need and effectiveness.

8. Use local intelligence and teamwork to generate greater knowledge and innovation, and use local analysis (epidemiology of differences) to present evidence for policy change.

9. At the national level, maintain rigor but introduce more flexibility: guidelines rather than norms.

10. Introduce measures to shorten the interim between research results and application, with the understanding that saving time also saves lives. -1 -

\section{References \& Notes}

1. Anuario Estadístico de Salud 2007. Havana: Ministry of Public Health, National Statistics Division; 2008.

2. Programa Integral para el Control del Cáncer en Cuba: Pautas para la Gestión. Vol. 1. Havana: Ministry of Public Health, National Cancer Control Unit; 2009.

3. Ministry of Public Health, National Statistics Division, Havana, data cited for 2007.

4. Segunda Encuesta Nacional de Factores de Riesgo de Enfermedades Crónicas no Transmisibles en Cuba. Informe de Trabajo. Havana: National Institute of Hygiene, Epidemiology and Microbiology; 2002.

5. Gaceta Oficial de la República de Cuba. Resolución Ministerial No. 49, 5 Jul 2006, p. 688-90. 